\title{
Strong Dynamical Heterogeneities in the Violation of the Fluctuation-Dissipation Theorem in Spin Glasses
}

\author{
F. Romá, ${ }^{1,2}$ S. Bustingorry, ${ }^{1}$ P. M. Gleiser, ${ }^{1}$ and D. Domínguez ${ }^{1}$ \\ ${ }^{1}$ Centro Atómico Bariloche, 8400 San Carlos de Bariloche, Río Negro, Argentina \\ ${ }^{2}$ Departamento de Física, Universidad Nacional de San Luis, 5700 San Luis, Argentina
}

(Dated: October 2, 2018)

\begin{abstract}
We analyze numerically the violation of the fluctuation-dissipation theorem (FDT) in the $\pm J$ EdwardsAnderson (EA) spin glass model. Using single spin probability densities we reveal the presence of strong dynamical heterogeneities, which correlate with ground state information. The physical interpretation of the results shows that the spins in the EA model can be divided in two sets. In $3 \mathrm{D}$, one set forms a compact structure which presents a coarsening-like behavior with its characteristic violation of the FDT, while the other asymptotically follows the FDT. Finally, we compare the dynamical behavior observed in 3D with 2D.
\end{abstract}

PACS numbers: 75.10.Nr, 75.40.Gb, 75.40.Mg

The study of glassy behavior, characterized by out-ofequilibrium dynamics that present very long relaxation times, involves a vast range of different systems such as spin glasses [1], structural glasses, colloidal and polymeric systems, and granular systems to name just a few [2]. These systems present out-of-equilibrium properties such as aging and violation of the fluctuation-dissipation theorem (FDT) [3, 4], whose characterization can be used as signatures of typical dynamical behaviors. For example, according to their deviation from the FDT they can be classified into three different groups: coarsening, structural glass and spin glass systems [3]. These cases have been theoretically described in terms of replica-symmetry breaking (RSB): in coarsening systems replica symmetry is unbroken, structural glasses correspond to one-step RSB and spin glasses to full RSB [3]. In the case of short-range spin glasses, most results on the violation of the FDT have been qualitatively and quantitatively described in the framework of mean field theory (full RSB) [3, 5]. Also direct experimental evidence on the violation of the FDT in an insulating spin glass [6] have been fitted by mean field theory.

In this work we show that, in contrast to these results, the violation of the FDT in the $3 \mathrm{D} \pm J$ Edwards-Anderson (EA) model is the result of two components with completely different behaviors: one that tends to satisfy the FDT relation, and another which presents a violation of this relation similar to coarsening systems. The behavior of the latter component seems to be compatible with the droplet picture scenario [7].

We reveal the presence of two components in the violation of the FDT by a careful analysis of dynamical heterogeneities. In principle, two different approaches may be used to study the development of dynamical heterogeneities. On one hand, it is possible to use space or time coarse-grained protocols [8, 9]. On the other hand, it is possible to use single spin observables looking for a direct observation of the local heterogeneities. In any of these approaches, if one is interested in making disorder averages and chooses to identify the spins by their position in the lattice, trivial results are obtained, since the difference between spins are washed out [10]. Here we take a local approach, but in contrast with pre- vious works, we properly include disorder averages by using a constrained structure of the ground state called backbone (see below), which allows for much insight in the analysis of dynamical heterogeneities [11].

We begin our analysis by using single spin probability densities to show that dynamical heterogeneities are present in the violation of FDT in the $3 \mathrm{D} \pm J$ EA model. These heterogeneities present a non-trivial complex structure with bimodal distributions [11, 12, 13], and we refer them as strong, in contrast to weak dynamical heterogeneities which present unimodal distributions with an elongated shape. Then, as done in 2D in [11], we use information from the ground state topology to establish a quantitative relation between spatial and dynamical heterogeneities. This analysis allows us to present a new physical interpretation of the violation of FDT. Finally we compare the violation of FDT in $2 \mathrm{D}$ and $3 \mathrm{D}$, which reveals fundamental differences.

We consider the EA model for spin glasses [1], defined on a $D$-dimensional lattice of linear size $L$ with periodic boundary conditions. The Hamiltonian of the model is

$$
H=\sum_{\langle i, j\rangle} J_{i j} \sigma_{i} \sigma_{j}
$$

where $\sigma_{i}= \pm 1$ is the spin variable and $\langle i, j\rangle$ indicates a sum over the $2 D$ nearest neighbors. The coupling constants $J_{i j}=$ $\pm J$ are random variables chosen from a symmetric bimodal distribution. The time evolution of the model is governed by a standard Glauber dynamics with sequential random updates using a continuous time Monte Carlo algorithm [14].

In order to investigate the violation of the FDT we use a two-times protocol which emphasizes the out-of-equilibrium character of the dynamics. The initial condition corresponds to a quench from $T=\infty$ to the temperature of interest $T<$ $T_{c}=1.12[15]$ at $t=0$, where each spin takes a random value $\sigma_{i}= \pm 1$. From this initial condition different two-times quantities are analyzed, which depend on both the waiting time $t_{w}$, when the measurement begins, and a given time $t>t_{w}[3,4]$.

We focus on the single spin two-times correlation function, defined as $\mathscr{C}_{i} \equiv\left\langle C_{i}\left(t, t_{w}\right)\right\rangle=\left\langle\sigma_{i}(t) \sigma_{i}\left(t_{w}\right)\right\rangle$, with $t>t_{w}$, and where $\langle\ldots\rangle$ is an average over thermal histories, that is over 
different initial conditions and realization of the thermal noise. In terms of the single spin correlation, the global two-times correlation function can be expressed as

$$
C=\left[\frac{1}{N} \sum_{i=1}^{N} \mathscr{C}_{i}\right]_{a v}=\left[\frac{1}{N}\left\langle\sum_{i=1}^{N} \sigma_{i}(t) \sigma_{i}\left(t_{w}\right)\right\rangle\right]_{a v}
$$

where $N=L^{D}$ and $[\ldots]_{a v}$ indicates average over different realizations of bond disorder (samples). In the same way the global integrated response function is defined in term of the single spin integrated response function as

$$
\chi=\left[\frac{1}{N} \sum_{i=1}^{N} \chi_{i}\right]_{a v}=\left[\frac{1}{N h}\left\langle\sum_{i=1}^{N} \sigma_{i}(t) \operatorname{sign}\left(h_{i}\left(t_{w}\right)\right)\right\rangle\right]_{a v}
$$

where $\chi_{i} \equiv\left\langle\sigma_{i}(t) \operatorname{sign}\left(h_{i}\left(t_{w}\right)\right) / h\right\rangle$ and, as usually, a random field of intensity $h$ is switched on at time $t_{w}$ [3, 4]. All the results presented correspond to $T=0.8, h=0.1$ and $10^{4}$ thermal histories for each sample.

Using the global quantities defined in Eqs. (2) and (3), the out-of-equilibrium fluctuation-dissipation relation can be written as

$$
T \chi\left(t, t_{w}\right)=X(C)\left[1-C\left(t, t_{w}\right)\right]
$$

where $X$ is the fluctuation-dissipation ratio (FDR) [16]. A useful representation of Eq. (4) is the parametric plot of $T \chi$ vs. $C[3,4]$. When the FDT holds the FDR is $X=1$ and the parametric plot shows a linear relation with unitary slope. In an out-of-equilibrium situation the FDT does not longer hold and two regimes are observed: for $t / t_{w} \ll 1$ the system shows quasi-equilibrium with $X=1$, while for $t / t_{w} \gg 1$ a violation of the FDT is observed with $X<1$. The behavior of $X(C)$ for $t / t_{w} \gg 1$ allows for a simple classification of out-ofequilibrium systems into three main categories: $(i)$ the value $X=0$ is related to coarsening systems, (ii) a constant $X<1$ value is associated with structural glasses, and (iii) a decreasing monotonic $0<X(C)<1$ function is associated to spin glasses. We will later use this simple scheme to physically reinterpret the out-of-equilibrium dynamics of spin glasses. In particular, the continuous line in Fig. 1 shows the parametric plot for the 3D EA model with linear size $L=20$ for $t_{w}=10$. The results correspond to averages over 50 different samples. After the departure from the quasi-equilibrium regime a continuous variation of the FDR is observed [12, 17].

In order to study how the heterogeneities arise in the violation of the FDT, we measure the joint probability distribution (JPD) of the single spin quantities, $\rho\left[\mathscr{C}_{i}\left(t, t_{w}\right), T \chi_{i}\left(t, t_{w}\right)\right]$. In Fig. 1 we show the projection of $\rho$ in the $(T \chi, C)$ plane for the same 50 samples used above. The sequence shows the evolution of the JPD for increasing $\Delta t=t-t_{w}$ as a map plot. The parametric plot (solid curve) of the global quantities is indicated in each figure as a guide to the eye, and for each value of $\Delta t$ the mean value of the distribution is indicated with a white circle.

Note that already for very short $\Delta t$ the distribution presents an elongated shape indicating an heterogeneous behavior. As
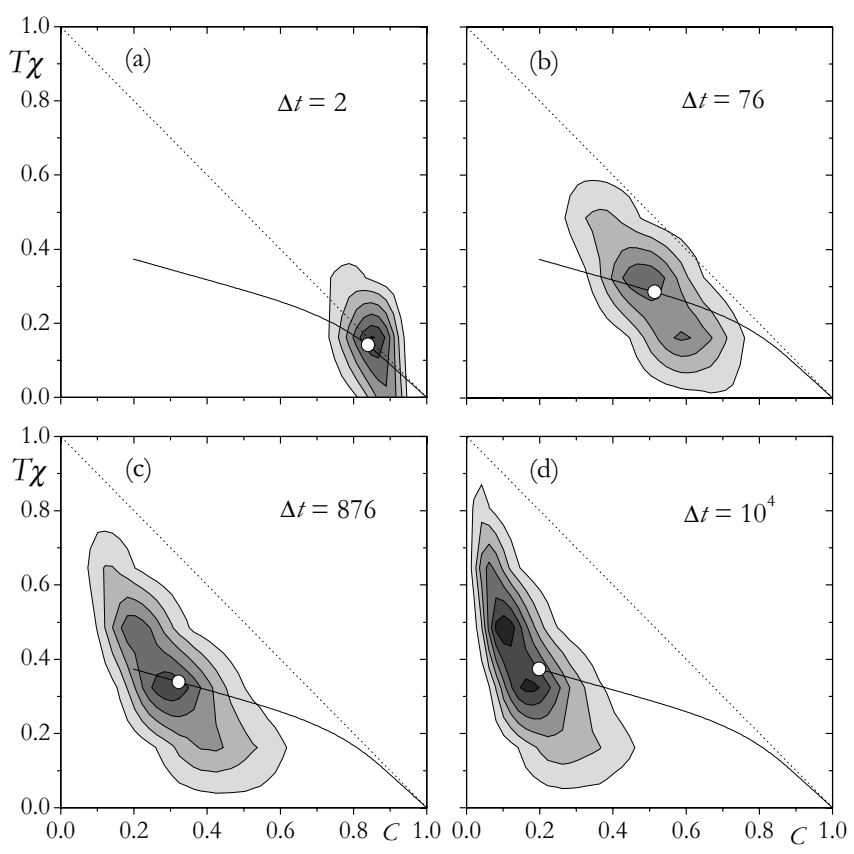

FIG. 1: Projection of the JPD of single spin correlations and integrated responses, $\rho\left[\mathscr{C}_{i}\left(t, t_{w}\right), T \chi_{i}\left(t, t_{w}\right)\right]$, for $L=20$ and $t_{w}=10$. The parametric plot of the global quantities, $T \chi(C)$, is reproduced in all the figures as a continuous line. The white dot indicates the mean value of the distribution for each $\Delta t$.

$\Delta t$ grows the distribution elongates further and the presence of two peaks can be clearly observed. At this point, it is clear that the mean value of the bimodal distribution shown in Fig.1(d) cannot be used as a representative quantity to characterize the parametric plot. Since for the development of the two peaks it is necessary to reach the longest $\Delta t$ as possible, we chose to work with a low $t_{w}=10$ value. For longer $t_{w}$ values we observed the same trend to a bimodal JPD for long time differences.

In the search for the origin of these strong dynamical heterogeneities we focus our analysis on a constrained structure, which is usually called a backbone. The presence of a backbone in models defined on randomly connected graphs leads to time-scale separation and heterogeneous dynamics at sufficiently low temperatures [18]. A backbone is also present in the EA model [19, 20], which is formed by those bonds which are always satisfied or always frustrated in all groundstate configurations. This structure can be used to divide the spins in the system in two sets: solidary spins, which are the spins in the backbone and thus maintain their relative orientation in all the ground-state configurations, and non-solidary spins which are simply all the spins that are not in the backbone [11]. Further insight in the static properties of the 3D and 2D EA model has been recently addressed by Romá et al. [21, 22]. They found that the fraction of solidary spins in $3 \mathrm{D}(2 \mathrm{D})$ is $0.76(0.67)$ of the total. In contrast with what is observed in 2D [20, 21], the largest component of the solidary spins in 3D does not fragment as the system size grows, 

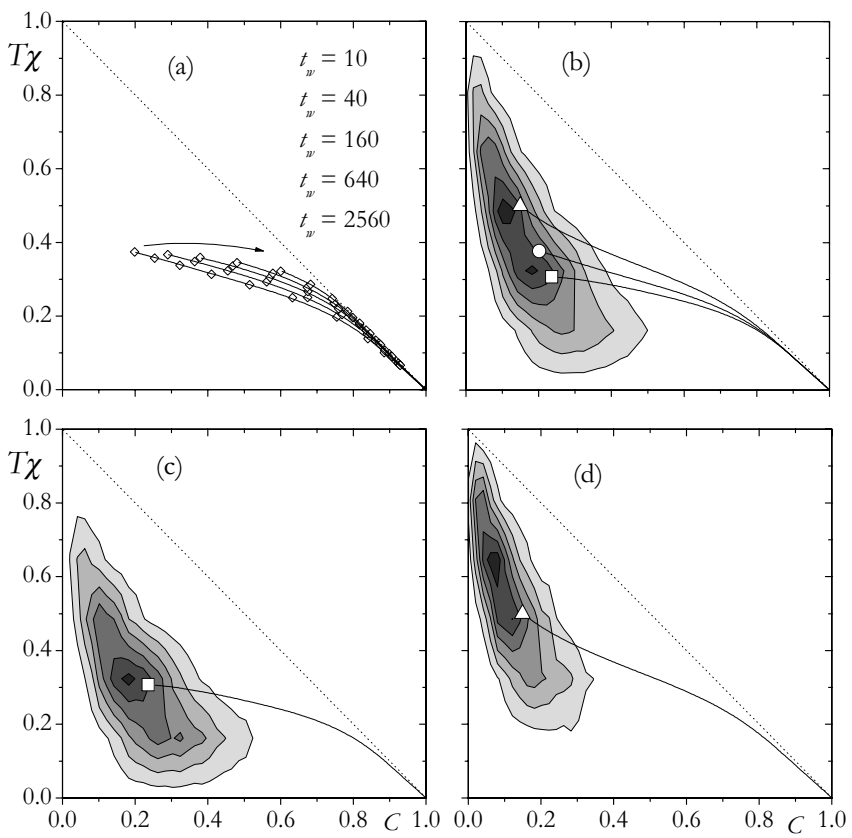

FIG. 2: (a) Parametric plot for $L=8$ (continuous line) and $L=20$ (open diamond) for five different waiting times. The arrow indicates increasing $t_{w}$. (b) Projection of $\rho$ and parametric plot of the global quantities $T \chi(C)$ for $L=8$ (circle). We also present the separation into sets A (square) and B (triangle). (c) and (d) show $\rho$ and its corresponding parametric plot constrained to sets $\mathrm{A}$ and $\mathrm{B}$ respectively. In (b), (c) and (d), the white symbols indicate the mean value of the JPD distribution for $t_{w}=10$ and $\Delta t=10^{4}$.

involving $60 \%$ of all the spins. Furthermore, the largest component in 3D forms a compact percolating cluster [22]. We use this information to analyze the strong dynamical heterogeneities observed in Fig. 1, and divide the system in two sets: the spins belonging to the percolating cluster of solidary spins (set A) and the remaining ones (set B).

Since we are interested in performing averages over disorder using this division, a considerable number of different realizations, where ground-state information is used, need to be taken into account. In order to calculate the backbone for each sample, we used an improvement of the Rigid Lattice Searching Algorithm as in [22]. This requires a large computational effort, since in order to obtain the exact backbone it is necessary to reach the ground state $O(N)$ times. This sets a limit to the largest size we can consider to $N=8^{3}$ [22].

To establish finite size effects we compare the results obtained with $L=8$ (over 300 samples) and $L=20$ (over 50 samples). In Fig. 22 a) we show the parametric plot for $L=8$ (continuous line) and $L=20$ (open diamond) for five different waiting times. For both system sizes the results overlap, which shows that, up to the times considered, no significant deviations due to finite size effects are present. Note also the shape of the JPD for $L=8$ (Fig. 22 b)) and $L=20$ (Fig. 11d)), which shows that for both system sizes the qualitative results obtained are similar.

Figure 2(b) also shows the behavior of the global paramet- ric plot (circle) and its division into sets $\mathrm{A}$ (square) and $\mathrm{B}$ (triangle). The JPD constrained to sets A and B are presented in Figs. 2(c) and 2 d) respectively. Note that the mean value of the JPD for the set A (B) is very close to the lower (upper) peak of the total JPD. In both cases the distributions are very broad, however the contribution of each set to the total distribution is clearly divided. Spins in set A (B) mainly contribute to the lower (upper) peak.

We now present and support a simple physical interpretation to understand the different behavior of each set of spins. Let us first highlight a significant topological property of set A. We observe that in average approximately $10 \%$ of the bonds in this set are frustrated in the ground state. By using a gauge transformation for a particular sample, which leaves the Hamiltonian invariant [23], one can change the bonds in set A so that all its spins are aligned in the ground state. As a consequence, this set is mapped into a ferromagnet with a low concentration $(\approx 10 \%)$ of anti-ferromagnetic bonds. Note that, when the anti-ferromagnetic bond concentration $x$ is changed in the 3D EA model, it is well established that a ferromagentic order is present below $x=0.22$ [24]. Then, since set A is below this limit $(x \approx 0.1)$, we expect a ferromagnetic-like order to persist in this region up to a finite temperature. This result allows us to establish an unexpected property of the $3 \mathrm{D} \pm J$ EA model: if restricted to set $\mathrm{A}$, an out-of-equilibrium behavior in accordance with that observed in a coarsening system should be found.

In order to analyze the violation of the FDT, we present in Fig. 3 (a) the parametric plot for each set for ten different $t_{w}$. We find that the lower curves, which correspond to set A, present a clear saturation to a curve away from the FDT line for increasing $t_{w}$, which is not observed when the system is considered as a whole (Fig. 3 (b)). This behavior reflects the particular topological characteristics of set A. We expect that for larger system sizes and long times the curves will follow an out-of-equilibrium behavior similar to the violation of the FDT in coarsening systems [3].

In contrast to the saturation behavior of set $\mathrm{A}$, the upper curves in Fig. 3 (a) show that set B tends to the FDT limit for increasing $t_{w}$. This surprising result is completely unexpected, since the out-of-equilibrium dynamics of the system is taking place below the critical temperature $T_{c}$, and a violation of the FDT should be observed when starting from random initial conditions $\left(T \gg T_{C}\right)$ [2, 3]. Notice that all the information on the underlying processes, which we observe using the division into sets $\mathrm{A}$ and $\mathrm{B}$, is lost when one considers the system as a whole (Fig. 3 (b)).

In order to stress the fundamental differences that can be pointed out in the intuitive frame proposed, we compare the out-of-equilibrium dynamics of the EA model in 2D and 3D. These systems present very similar behavior at low temperatures, allowing for comparisons between their dynamics [11, 18, 25]. However, the fact that $T_{c}=0$ in $2 \mathrm{D}$ and $T_{c}>0$ in $3 \mathrm{D}$, recalls that the physical mechanisms present in the dynamics are clearly different. Notice also that since there is no percolating cluster of solidary spins in 2D [20], the system can 

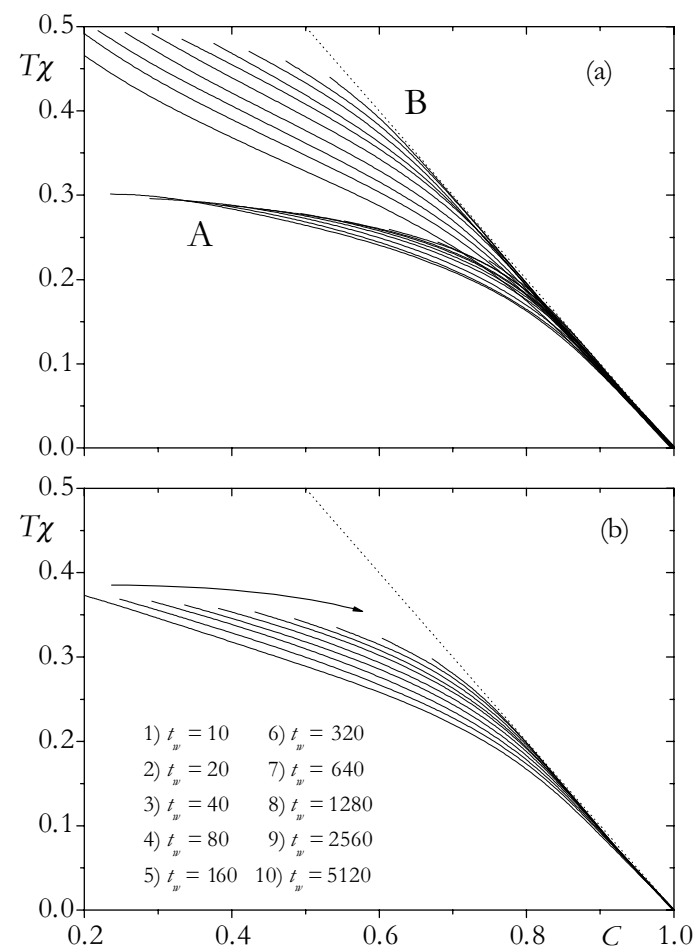

FIG. 3: Parametric plot for: (a) sets A and B and (b) for the system as a whole. The arrow indicates increasing $t_{w}$ for the ten different waiting times.

only be divided in solidary and non-solidary spins [11]. Using this division for the 2D EA model we found that, as expected, the evolution of the parametric plot for both sets tends to the FDT limit for increasing $t_{w}$, in contrast to the results obtained in $3 \mathrm{D}$.

Summarizing, we observe strong dynamical heterogeneities in the JPD of single spin correlation and integrated response functions. We have gone beyond previous works on single spin quantities, and have been able to show that these heterogeneities persist even when averages over disorder are taken into account. Using information from the ground state topology we are able to establish a quantitative relation between spatial and dynamical heterogeneities. In particular, we observe that in the 3D EA model there is a fraction of the system which is capable of sustaining a ferromagnetic-like order. The presence of this structure is reflected in the parametric plot of $T \chi$ vs. $C$. On the other hand, the other fraction of the system presents a dynamical behavior that asymptotically tends to satisfy the FDT. As a consequence, the global outof-equilibrium dynamics of the system can be regarded as a combination of two different components. A comparison of the violation of the FDT in 3D with 2D reveals that the underlying physical mechanisms present in the dynamics are clearly different.

We have shown that the continuous violation of the FDT, considered a signature of full RSB, can be regarded as the combination of two different components. This particular re- sults suggest that in short-range spin glasses the droplet picture could be valid if restricted to a finite fraction of the system. However, as we have shown in the 3D EA model, to understand the behavior of the system as a whole it is also neccesary to take into account the existence of a finite fraction of the system which presents a paramagnetic behavior. We believe that the inclusion of these elements in a new theoretical description can contribute to the understanding of the physical nature of spin glasses. In particular it can contribute to the study of the relation between the percolation problem and the spin-glass transition [26], and also to hot topics such as temperature and disorder chaos [27].

We thank L.F. Cugliandolo, J. Kurchan and S. RisauGusman for fruitful discussions. We acknowledge financial support from CNEA and CONICET (Argentina), and ICTP NET-61 (Italy). F.R., S.B and D. D acknowledge support from CONICET, PIP05-5596. F.R. thanks Univ. Nac. de San Luis (Argentina) project 322000 and Millennium Scientific Iniciative (Chile) contract P-02-054-F for partial support. P.M.G. acknowledges financial support from CONICET PIP05-5114 (Argentina) and ANPCyT PICT03-13893 (Argentina).

[1] M. Mezard, G. Parisi, and M. A. Virasoro, Spin Glass Theory and Beyond, (World Scientific, Singapore, 1987).

[2] Slow Relaxations and Nonequilibrium Dynamics in Condensed Matter, edited by J.-L. Barrat et al. (Springer, Berlin, 2002).

[3] A. Crisanti and F. Ritort, J. Phys. A: Math. Gen. 36, R181 (2003).

[4] L. F. Cugliandolo in Ref. [2].

[5] S. Pérez Gaviro, J. J. Ruiz-Lorenzo, and A. Tarancón, J. Phys. A: Math. Gen. 39, 8567 (2006).

[6] D. Herisson and M. Ocio, Phys. Rev. Lett. 88, 257202 (2002).

[7] D. S. Fisher and D. A. Huse, Phys. Rev. Lett. 56, 1601 (1986).

[8] H. E. Castillo et al., Phys. Rev. B 68, 134442 (2003).

[9] C. Chamon et al., J. Chem. Phys. 121, 10120 (2004).

[10] A. Montanari and F. Ricci-Tersenghi, Phys. Rev. Lett. 90, 017203 (2003); Phys. Rev. B 68, 224429 (2003).

[11] F. Romá, S. Bustingorry, and P. M. Gleiser, Phys. Rev. Lett. 96, 167205 (2006).

[12] F. Ricci-Tersenghi and R. Zecchina, Phys. Rev. E 62, R7567 (2000).

[13] Y. Jung, J. P. Garraham, and D. Chandler, J. Chem. Phys. 123, 084509 (2005).

[14] A. B. Bortz, M. H. Kalos, and J. L. Lebowitz, J. Comp. Phys. 17, 10-18 (1975).

[15] H. G. Katzgraber, M. Koerner, and A. P. Young, Phys. Rev. B 73, 224432 (2006).

[16] L. F. Cugliandolo, J. Kurchan, and L. Peliti, Phys. Rev. E 55, 3898 (1997).

[17] A. Barrat, Phys. Rev. E 57, 3629 (1998).

[18] A. Barrat and R. Zecchina, Phys. Rev. E 59, R1299 (1999).

[19] J. Vannimenus, J. M. Maillard, and L. de Sèze, J. Phys. C: Solid State Phys. 12, 4523 (1979).

[20] F. Barahona, R. Maynard, R. Rammal, and J.P. Uhry, J. Phys. A: Math. Gen. 15, 673 (1982).

[21] F. Romá et al., Physica A (Amsterdam) 363, 327 (2006).

[22] F. Romá et al., Phys. Rev. B 75, 020402(R) (2007). 
[23] G. Toulouse, Commun. Phys. 2, 115 (1977).

[24] A. K. Hartmann, Phys. Rev. B 59, 3617 (1999).

[25] A. Barrat and L. Berthier, Phys. Rev. Lett. 87, 087204 (2001).

[26] D. Stauffer, J. Phys. A: Math. Gen. 26 L525-L528 (1993).
[27] H. G. Katzgraber and F. Krza̧kała, Phys. Rev. Lett. 98, 017201 (2007). 